\title{
Kondo Lattice and Magnetic Properties in Strongly Correlated Electron Systems
}

\author{
B. CoqBlin* \\ Laboratoire de Physique des Solides (UMR 8502), CNRS, Université Paris-Sud \\ 91405 Orsay, France
}

\begin{abstract}
We review here the magnetic properties of some strongly correlated electron systems and especially cerium and uranium compounds. The normal Kondo lattice model with a localized $S=1 / 2$ spin can account for the Kondo-magnetism competition observed in cerium or ytterbium systems, while the underscreened Kondo lattice model with a localized $S=1$ spin can well account for the ferromagnetism - Kondo coexistence observed in some uranium compounds such as UTe. Then, we discuss the spin glass-Kondo competition and present the resulting phase diagrams showing spin glass, Kondo and magnetically ordered phases observed in disordered heavy fermion cerium alloys such as $\mathrm{CeNi}_{x} \mathrm{Cu}_{1-x}$ alloys.
\end{abstract}

PACS numbers: 71.27.+a, 72.15.Qm, 75.10.Nr, 75.20.Hr

\section{Introduction}

It is well known that rare-earth metals are magnetic with a magnetic moment corresponding to the configuration $4 f^{n}$ with integer $n$ values, except the "anomalous" metals, cerium, europium and ytterbium metals. An anomalous behavior has been also observed in some alloys and compounds containing these three rare-earths, but also praseodymium, samarium and thulium. On the other hand, magnetism occurs only for curium in the middle of the series of the actinide metals, but many uranium, neptunium, plutonium and americium systems are known to be magnetic or to present an anomalous behavior [1-3].

Cerium systems, which present several different behaviors, have been extensively studied. First of all, the phase diagram of cerium metal is very interesting. At room temperature and normal pressure, cerium metal is in the $\gamma$ phase which is almost trivalent with a number of $4 f$ electrons very close to 1 and is magnetic with a magnetization corresponding to the $4 f^{1}$ configuration. When pressure is applied at room temperature, cerium undergoes a first-order transition from this $\gamma$ phase to the $\alpha$ phase which is no more magnetic and has an "intermediate valence" with a smaller number of $4 f$ electrons [4]. The first theoretical explanation was based on the "promotion" model and starts from a treatment of the spin and orbitally degenerate Anderson Hamiltonian, which gives a transition from a magnetic trivalent phase to a non-magnetic intermediate valence one [5]. Indeed, there have been several other theoretical explanations of the phase diagram of cerium, based on a magnetic transition within the promotion model [6] or on the Mott transi-

* e-mail: coqblin@lps.u-psud.fr tion [7] or also the "Kondo collapse" model [8, 9]; in this model, the effective Kondo temperature is taken to be much larger in the $\alpha$ phase than in the $\gamma$ phase and the effective valence equal to the number of conduction electrons remains relatively small of order 3.3 in the $\alpha$ phase. Thus, in spite of differences in the theoretical explanations, the phase diagram of cerium can be considered as a very good example of the difference between the magnetic case with an almost integer number of $4 f$ electrons $N_{f}$ and the "intermediate valence" case with an intermediate $N_{f}$ value. Moreover, the number of $4 f$ electrons is often not an integer and varies with the external pressure or the matrix concentration in many systems, like $\mathrm{Eu}\left(\mathrm{Pd}_{1-x} \mathrm{Au}_{x}\right)_{2} \mathrm{Si}_{2}$ alloys [10] or $\mathrm{YbInCu}_{4}$ [11].

On the other hand, Kondo [12] explains in 1964 the decrease in the magnetic resistivity in $\log T$ and, therefore, the minimum of the total electrical resistivity, by a perturbation calculation performed on the spin exchange Hamiltonian given by $J_{\mathrm{K}} s_{c} S_{f}$ in the case of an antiferromagnetic coupling with $J_{\mathrm{K}}>0$. The "Kondo effect" has been observed in many magnetic dilute alloys such as CuFe, LaCe, $\underline{\mathrm{AuYb}}$, and also in many compounds with cerium or ytterbium or with other anomalous rare-earths such as $\mathrm{PrSn}_{3}$ or TmS. Then, at very low temperatures, the single Kondo impurity has a heavy fermion behavior, characterized by very large values of the electronic specific heat constant $\gamma$ and of the coefficient of the $T^{2}$ law of the electrical resistivity and the localized magnetic moment $S_{f}=1 / 2$ is completely screened by the conduction electron spins $s_{c}=1 / 2$ at $T=0$.

The Kondo behavior corresponds to a number of $f$ electrons close to an integer and it is well known that we can separate at first sight the anomalous behaviors observed in rare-earth or actinide strongly correlated systems in either "intermediate valence" or "Kondo" ones. Here, 
we will review only the Kondo systems and we will discuss essentially the case of a Kondo lattice in strongly correlated electron systems.

Thus, we will review here briefly three main recent theoretical results on the normal Kondo lattice model with localized spins $S=1 / 2$ which can describe the competition between the Kondo effect and the magnetic ordering in cerium compounds $[13,14]$, then on the underscreened Kondo lattice (UKL) model with localized spins $S=1$ which can be applied to the Kondo-ferromagnetism coexistence observed in some uranium compounds [15] and finally on the competition between the Kondo effect and the spin glass in disordered cerium alloys [16].

\section{The Kondo lattice model}

In the regular Kondo problem, the spin $S_{f}=1 / 2$ of the localized $4 f$ electron (or hole) corresponding to the $4 f^{1}$ (or $4 f^{13}$ ) configuration is completely screened at very low temperatures by the spin $s_{c}=1 / 2$ of the conduction electrons. It is well known that, in the regular Kondo lattice, there is a competition between the Kondo effect and the magnetic ordering arising from the Ruderman-KittelKasuya-Yosida (RKKY) interaction between rare-earth atoms at different lattice sites, which has been well described by the Doniach diagram [17]. This diagram, shown in Fig. 1, gives the variation of the ordering tem-

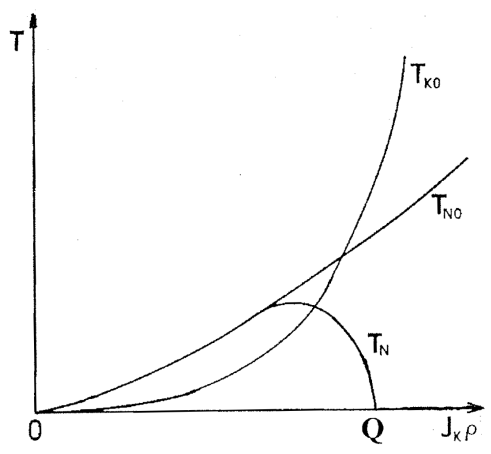

Fig. 1. The Doniach diagram giving different temperatures versus $\rho J_{\mathrm{K}}$. $T_{\mathrm{K} 0}, T_{\mathrm{N} 0}$ and $T_{\mathrm{N}}$ design respectively the single-impurity Kondo temperature, the corresponding Néel temperature and the Néel temperature for the lattice.

perature and of the Kondo temperature with increasing $J_{\mathrm{K}}$. The resulting ordering temperature $T_{N}$ of the lattice is increasing initially with increasing $\rho J_{\mathrm{K}}$, then passes through a maximum and tends to zero at a critical value $\rho J_{\mathrm{K}}^{\mathrm{c}}$ corresponding to a "quantum critical point" (QCP). Such a behavior of $T_{N}$ has been experimentally observed with increasing pressure in many cerium compounds, such as $\mathrm{CeAl}_{2}$ [18] or $\mathrm{CeRh}_{2} \mathrm{Si}_{2}$ [19]. A similar effect has been observed in $\mathrm{YbCu}_{2} \mathrm{Si}_{2}[20]$ or in other ytterbium compounds, where the Néel temperature starts from zero at a given pressure and increases rapidly with pressure, since pressure has an opposite effect in ytterbium compounds than in cerium compounds.
For $\rho J_{\mathrm{K}}$ values smaller than the value $\rho J_{\mathrm{K}}^{c}$ of the quantum critical point $Q$, cerium compounds are magnetically ordered with a small heavy fermion character and with relatively small ordering temperatures, typically of order $5-10 \mathrm{~K}$. For $\rho J_{\mathrm{K}}$ values larger than $\rho J_{\mathrm{K}}^{c}$, cerium compounds are non-magnetic and can have a very large heavy fermion character with a $\gamma$ typically of order $1 \mathrm{~J} /\left(\mathrm{mol} \mathrm{K}^{2}\right)[1]$.

The Kondo lattice model has been studied in detail within the mean-field approximation [14]. We have introduced a Kondo lattice model with both an intrasite Kondo exchange interaction and an intersite magnetic exchange interaction between neighboring localized spins and the resulting Hamiltonian is, therefore, given by

$$
\begin{aligned}
H & =\sum_{\boldsymbol{k} \sigma} \varepsilon_{\boldsymbol{k}} n_{\boldsymbol{k} \sigma}^{c}+\sum_{i \sigma} E_{0} n_{i \sigma}^{f}+J_{\mathrm{K}} \sum_{i} \boldsymbol{s}_{i} \cdot \boldsymbol{S}_{i} \\
& +J_{H} \sum_{\langle i j\rangle} \boldsymbol{S}_{i} \cdot \boldsymbol{S}_{j},
\end{aligned}
$$

where $\varepsilon_{\boldsymbol{k}}$ is the energy of the conduction band, $J_{\mathrm{K}}(>0)$ is the Kondo coupling between the localized spin $\boldsymbol{S}_{i}$ and the spin $\boldsymbol{s}_{i}$ of a conduction electron at the same site and $J_{H}$ is the interaction between nearest-neighboring localized spins. We consider here the case of choosing $J_{H}$ to be positive, implying that intersite interactions are antiferromagnetic, as it is the case of most cerium compounds.

Then, in all these methods used to describe both the Kondo effect and either short-range magnetic correlations or a given magnetic order (which could be antiferromagnetic, ferromagnetic or spin glass ...), we use two types of operators, describing the Kondo correlations or the magnetic correlations. In our first study [13], we have used a mean-field approximation with two correlators, $\lambda$, describing the intrasite Kondo correlation, and $\Gamma$, representing an intersite correlation between two neighboring moments

$$
\lambda=\left\langle c_{i \sigma}^{+} f_{i \sigma}\right\rangle, \quad \Gamma=\left\langle f_{i \sigma}^{+} f_{j \sigma}\right\rangle .
$$

Here, we summarize briefly the magnetism-Kondo competition and we will discuss here the problem of "exhaustion" principle which has been introduced by Nozières [21]. Detailed calculations are presented in Refs. $[13,14]$. In the mean field approximation, the Kondo temperature $T_{\mathrm{K}}$ and the correlation temperature $T_{\text {cor }}$ are defined as the temperatures at which, respectively, $\lambda$ and $\Gamma$ become zero. We have studied the existence of the Kondo phase (with $\lambda$ different from zero) and of the short-range magnetic phase (with $\Gamma$ different from zero), as a function of the two parameters $J_{\mathrm{K}}$ and $J_{H}$, but also of the number $n_{c}$ of conduction electrons. Figure 2 shows the effect of the different parameters: $J_{\mathrm{K}}$ tends to favor the Kondo effect, while $J_{H}$ tends to favor magnetism and is opposite to the Kondo effect. Moreover, the Kondo temperature and the Kondo phase decrease when $n_{c}$ decreases, as clearly shown in Fig. 2. In this figure, we have plotted the curves derived by analytical calculations, while the points correspond to the results obtained numerically by minimizing the energy and we see clearly 


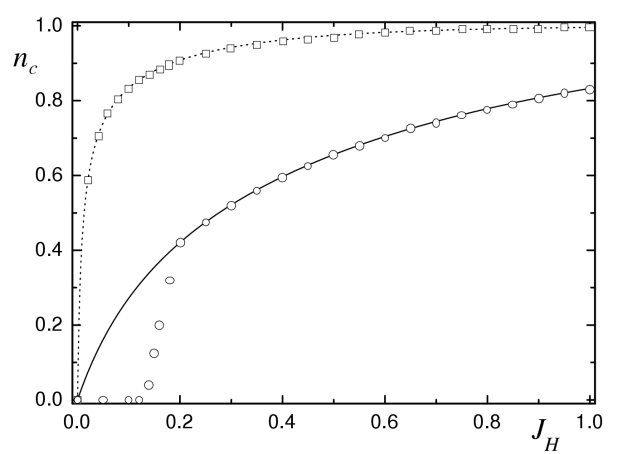

Fig. 2. Phase diagram between the Kondo and the magnetic phases, versus the number $n_{c}$ of conduction electrons and the intersite exchange $J_{H}$ for $J_{\mathrm{K}}=0.4$ (dotted line) and $J_{\mathrm{K}}=1$ (solid line). The lines correspond to analytical calculations and the points to numerical ones obtained by minimizing the energy. In each case, the Kondo regime is above the line and the magnetic phase below, according to Ref. [14].

that the increase in $J_{H}$ and the decrease in $n_{c}$ tend to decrease the Kondo effect, as explained in Ref. [14].

Then, the temperature $T_{\text {cor }}$ for the occurrence of shortrange magnetic correlations lies above the Kondo temperature $T_{\mathrm{K}}$ for large $J_{H}$ values, while $T_{\text {cor }}=T_{\mathrm{K}}$ for small $J_{H}$ values. The theoretical result giving a temperature $T_{\text {cor }}$ larger than $T_{\mathrm{K}}$ has been observed by neutron scattering experiments in some cerium compounds [22]. It has been also established, for $n_{c}=1$ [13] and then for $n_{c}<1$ [14], that the Kondo temperature for the lattice can be significantly different and in fact generally smaller than the single-impurity one. The interpretation of the experimental results is not easy, but it was suggested experimentally in some cerium compounds, like $\mathrm{CeRh}_{2} \mathrm{Si}_{2}$ [19], that the Kondo temperature is smaller than the single impurity Kondo temperature $T_{\mathrm{K} 0}$ and does not present an exponential variation as expected for $T_{\mathrm{K} 0}$. Further experiments are needed to better understand the conditions yielding a Kondo temperature for the lattice different from the single-impurity one.

Finally, it is important to notice that ytterbium compounds do not present a "mirror" behavior just opposite to that of cerium compounds [23]. We can argue that the $4 f$ electrons are more localized in ytterbium than in cerium, which results in smaller $J_{H}$ values for $\mathrm{Yb}$ and this idea can explain the fact that $\mathrm{Yb}$ compounds have a different behavior.

\section{The underscreened Kondo lattice model}

The situation of actinide systems is complex and different from that of rare-earth systems, because the $5 f$ electrons are clearly in a crossover region between localized and itinerant behavior and are any way less localized than the $4 f$ electrons of rare-earths, as seen for example in the series of actinide metals where magnetism occurs only for curium in the middle of the series. It is well known that uranium compounds can present a great variety of different behaviors, from magnetic ones to non-magnetic ones with for example a possible spin fluctuation description of the $5 f$ electrons.

We will discuss here only our recently developed UKL model [15] which can account for the coexistence between the Kondo effect and the ferromagnetic ordering which has been observed in some uranium compounds.

Uranium compounds, UTe [24, 25], $\mathrm{UCu}_{0.9} \mathrm{Sb}_{2}[26]$ or $\mathrm{UCo}_{0.5} \mathrm{Sb}_{2}[27]$ present a ferromagnetic ordering at very large Curie temperatures (equal respectively to $T_{\mathrm{c}}=$ $102 \mathrm{~K}, 113 \mathrm{~K}$ and $64.5 \mathrm{~K}$ ) and show also a $\log T$ Kondo-type decrease in the resistivity above $T_{\mathrm{c}}$. A similar behavior has been recently observed in the neptunium compound $\mathrm{NpNiSi}_{2}[28]$ and even also in the $d$-electron ferromagnetic compound $\mathrm{Yb}_{14} \mathrm{MnSb}_{11}$ [29].

Uranium monochalcogenides provide a good example of the complexity of the $5 f$ electrons, which appear to be itinerant in US, localized in UTe and in between in USe $[25,30]$. It is difficult to interpret different experiments in UTe, but clearly the magnetic moments deduced from magnetic susceptibility experiments in this compound are close to the free ion values of uranium, which implies that the $5 f$ electrons are relatively well localized in UTe $[25,31]$ and can be correctly described within a $5 f^{2}$ configuration.

In the UKL model [15], the two $5 f$ electrons are bound into a spin $S=1$. The Hamiltonian is the same as that of Eq. (1), except two essential differences: First, the localized spins are here $\boldsymbol{S}=1$ and second, the term $\sum_{i \sigma \alpha} E_{0} n_{i \sigma}^{f_{\alpha}}$ describing the energy of localized electrons at the energy $E_{0}$ contains now a sum on the two $5 f$ electrons (defined by the notation $\alpha=1,2$ ) to describe the $5 f^{2}$ configuration of uranium atoms. Finally, the intersite interaction is ferromagnetic with $J_{H}<0$.

In the UKL model of Ref. [15], we have used the mean field approximation by introducing the operators

$$
\widehat{\lambda}_{i \sigma}=\sum_{\alpha} \widehat{\lambda}_{i \sigma}^{\alpha}=\sum_{\alpha}\left\langle c_{i \sigma}^{+} f_{i \sigma}^{\alpha}\right\rangle,
$$

which couples conduction and $f$ electrons at the same site.

This method is able to give a good description of the Kondo effect. Moreover, in order to describe the magnetic properties of the system, we introduce the magnetization operators for both $f$ and $c$ electrons

$$
\begin{aligned}
& M_{i}=S_{i}^{z}=\frac{1}{2}\left(n_{i \uparrow}^{f}-n_{i \downarrow}^{f}\right), \\
& m_{i}=\sigma_{i}^{z}=\frac{1}{2}\left(n_{i \uparrow}^{c}-n_{i \downarrow}^{c}\right) .
\end{aligned}
$$

The non-zero values of $\langle M\rangle$ and $\langle m\rangle$ describe the magnetic phase with a non-zero total magnetization, while a non-zero $\lambda_{\sigma}$ describes the Kondo effect and the formation of the heavy-fermion state.

The detailed calculations can be found in Ref. [15] and we present here only the plots of the Kondo temperature $T_{\mathrm{K}}$ and of the Curie temperature $T_{\mathrm{c}}$ as a function of the Kondo coupling constant $J_{\mathrm{K}}$. The Kondo temperature 
$T_{\mathrm{K}}$ becomes finite only at the critical value $J_{\mathrm{K}}^{\mathrm{c}}$ of order 0.65 and then increases rapidly for larger values of $J_{\mathrm{K}}$. On the other hand, the Curie temperature, $T_{\mathrm{c}}$, is finite for all studied values of $J_{\mathrm{K}}$. The two curves of $T_{\mathrm{K}}$ and $T_{\mathrm{c}}$ cross, slightly above $J_{\mathrm{K}}^{\mathrm{c}}$ and, for larger values of $J_{\mathrm{K}}$, the Kondo temperature, $T_{\mathrm{K}}$, is always larger than $T_{\mathrm{c}}$.

The resulting "ferromagnetic-Kondo" diagram, shown in Fig. 3, is completely different from the Doniach diagram derived for the Kondo lattice model appropriate for cerium or ytterbium compounds and represents really a new result for the study of magnetic actinide compounds.

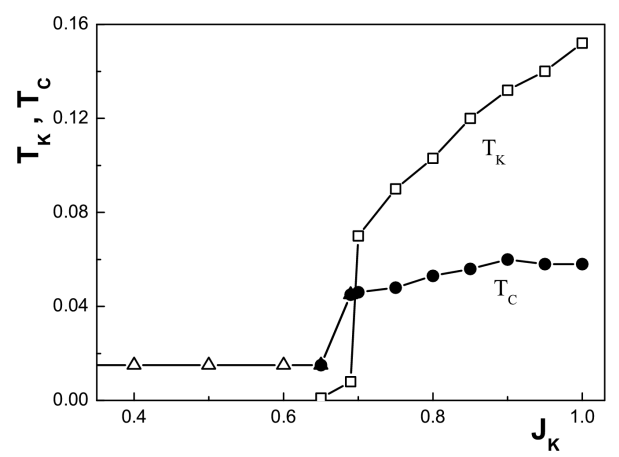

Fig. 3. Kondo-ferromagnetic phase diagram: plot of the Curie temperature $T_{\mathrm{c}}$ and the Kondo temperature $T_{\mathrm{K}}$ versus $J_{\mathrm{K}}$ for $J_{H}=-0.01$ and $n_{c}=0.8$, with the notations of Ref. [15].

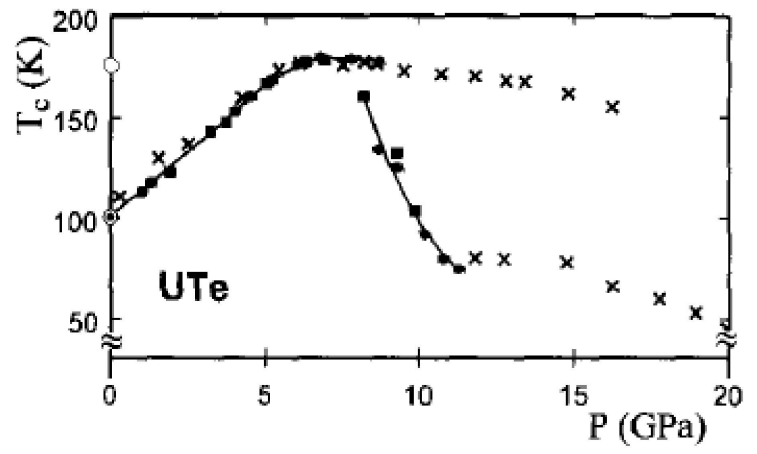

Fig. 4. Curie temperature $T_{\mathrm{c}}$ of UTe compound versus pressure. The black circles and squares come from Ref. [32] and the crosses from Ref. [33].

The ferromagnetic uranium monochalcogenides UTe, USe and US have been studied at very high pressures $[32,33]$. Figure 4 gives the experimental variation of the Curie temperature of UTe versus pressure, which presents an increase in $T_{\mathrm{c}}$ up to a maximum at roughly $7 \mathrm{GPa}$ and then a clear decrease with larger pressures, in spite of different experimental results $[32,33]$. On the opposite, the Curie temperature of US is continuously decreasing with pressure and $T_{\mathrm{c}}$ of USe remains constant up to 10 GPa and decreases rapidly at higher pressures [32].
The initial increase in $T_{\mathrm{c}}$ with pressure is clearly explained by our model, since Fig. 3 gives an increase in $T_{\mathrm{c}}$ versus $J_{\mathrm{K}}$ above the critical value $J_{\mathrm{K}}^{\mathrm{c}}$ and $J_{\mathrm{K}}$ increases with pressure. On the other hand, the maximum and the decrease in $T_{\mathrm{c}}$ at higher pressure cannot be understood in the UKL model and this decrease is directly connected to a decrease in the $5 f$ magnetization corresponding to a decrease in the number of $5 f$ electrons and to an effective delocalization of the $5 f$ electrons. Similarly, the decrease in $T_{\mathrm{c}}$ in US compound corresponds to a continuous delocalization of the $5 f$ electrons which are already itinerant at normal pressure. The case of USe is intermediate, with $5 f$ electrons between localized and itinerant at normal pressure. Sheng and Cooper [30] have performed ab initio band calculations which can account for the pressure dependence of $T_{\mathrm{c}}$ in uranium monochalcogenides.

Any way, our previous UKL model with a fixed total number $n_{f}=2$ of $5 f$ electrons has to be extended into a more general underscreened Anderson lattice (UAL) model which starts from two $f$ electrons, $f_{1 \sigma}$ and $f_{2 \sigma}$, but considers the possibility of the decrease in $n_{f}$ below 2 .

Work is presently in progress to study the UAL model within the Green function technique [34] and to obtain the variation of $T_{\mathrm{c}}$ with pressure and the departure from the UKL model in UTe at very high pressures.

\section{The Kondo-spin glass - magnetic order competition}

An extensive work has been also performed to study the competition between the Kondo effect, the spin glass and the ferromagnetic ordering which has been observed in disordered cerium alloys. The most studied case is the $\mathrm{CeNi}_{x} \mathrm{Cu}_{1-x}$ alloys $[35,36]$, but we can also cite the $\mathrm{Ce}_{2} \mathrm{Au}_{1-x} \mathrm{Co}_{x} \mathrm{Si}_{3}$ alloys [37] and some disordered uranium alloys such as $\mathrm{UCu}_{5-x} \mathrm{Pd}_{x}$ [38] or $\mathrm{U}_{1-x} \mathrm{La}_{x} \mathrm{Pd}_{2} \mathrm{Al}_{3}$ [39].

The $\mathrm{CeNi}_{x} \mathrm{Cu}_{1-x}$ alloys were extensively studied by firstly bulk experimental methods and then by microscopic measurements like $\mu \mathrm{SR}$ spectroscopy or neutron diffraction. These alloys are at low temperatures antiferromagnetic for low $\mathrm{Ni}$ concentration $(x$ small $)$ and have a Kondo behavior for low $\mathrm{Cu}$ concentration ( $x$ large), but the experimental situation is more complicated in the intermediate case, typically of $x$ between 0.3 and 0.7 . It was firstly shown that, when temperature decreases, there are successively a spin-glass phase and then a ferromagnetic one [40]. More recent experiments have been analyzed in terms of an inhomogeneous "cluster spin glass" or "cluster glass" (CG) followed at lower temperature by an inhomogeneous ferromagnetic (IFM) order according to neutron diffraction at very low temperature. However, there is no clear indication of a Curie temperature $T_{\mathrm{c}}$ from ac susceptibility and specific heat measurements. Recent experiments have confirmed the emergence of a spin glass-like state and a percolative evolution of the clusters from the CG state to the IFM one with decreasing temperature [36, 41]. 
On the other hand, there has been an extensive effort to understand the complicated competition between the Kondo effect, the spin glass and magnetic orderings in such disordered cerium alloys. The calculation starts from a Hamiltonian similar to that given by Eq. (1), with also an intrasite Kondo interaction term with an exchange integral $J_{\mathrm{K}}$ but with a different intersite interaction $H_{\mathrm{SG}}$ corresponding to the spin-glass phase

$$
H_{\mathrm{SG}}=\sum_{i, j} J_{i j} S_{f i}^{z} S_{f j}^{z} \text {. }
$$

In a first calculation, we have taken the SherringtonKirkpatrick model [42], where the exchange intersite integrals are assumed to be randomly distributed with a Gaussian distribution. The first model assumes a zero mean value of the Gaussian distribution in order to study only the Kondo-spin glass interaction and we have, therefore, obtained a phase diagram showing first a spin-glass phase and then a Kondo phase with increasing $J_{\mathrm{K}}$ [43]. Then, in order to obtain a more complex phase diagram with a ferromagnetic [44] or an antiferromagnetic [45] phase occurring at low temperatures for smaller $J_{\mathrm{K}}$ values, we have taken the same model but with a non-zero mean value $J_{0}$ of the Gaussian distribution. Thus, it results that the Kondo phase obtained for large $J_{\mathrm{K}}$ values is still there, but that there is a competition between the spin glass phase and the magnetic phase for smaller $J_{\mathrm{K}}$ values. However, in the ferromagnetic case, when $J_{\mathrm{K}}$ is typically of order $J_{0}$, we have obtained with decreasing temperature successively a ferromagnetic phase, then a mixed ferromagnetic-spin glass phase and finally a spin-glass phase. The evidence of a spin-glass phase at very low temperatures is in disagreement with experimental results previously described for $\mathrm{CeNi}_{x} \mathrm{Cu}_{1-x}$ alloys.

Then, we have considered another new approach which introduces a generalization of the Mattis model [46] and which represents an interpolation between ferromagnetism and a highly disordered spin glass. The detailed calculations can be found in Ref. [16] and here we start from the following simple version of the exchange integrals between spins on different sites $i$ and $j$, given by

$$
J_{i j}=\frac{1}{N} \sum_{\mu} J \xi_{i}^{\mu} \xi_{j}^{\mu},
$$

where the $\xi_{i}^{\mu}= \pm 1(\mu=1,2, \ldots, p ; i=1,2, \ldots, N)$ are independent random distributed variables, with an equal probability for $\xi_{i}^{\mu}$ to be equal to +1 or -1 . The critical parameter is here the ratio $a=p / N$, which gives an estimation of the relative importance of the ferromagnetic and spin glass phases for small $J_{\mathrm{K}}$ values; for large $J_{\mathrm{K}}$ values, the Kondo phase is always present.

For a small value of $a$, typically $a=0.04$, we have obtained a spin-glass phase, then a mixed ferromagneticspin glass phase and finally a ferromagnetic phase with decreasing temperature for a relatively small $J_{\mathrm{K}} / J$ ratio. This theoretical result accounts better for the experimental phase diagram of $\mathrm{CeNi}_{x} \mathrm{Cu}_{1-x}$ alloys, with in particular the ferromagnetic phase below the spin-glass phase at the lowest temperatures [16].
In fact, the experimental phase diagram of $\mathrm{CeNi}_{x} \mathrm{Cu}_{1-x}$ alloys gives a percolative transition with decreasing temperature from a cluster-glass state with ferromagnetic correlations to a disordered ferromagnetic state [36, 41]. Following Ref. [16], we can think that the more "local" description given by Eq. (6) seems to be more adequate here than the "average" description. A real improvement will be provided by trying to describe better the clusters which are yielding the CG and the IFM phases, but it is rather difficult to obtain clusters with intrasite Kondo interactions and both intra- and inter-cluster magnetic interactions [47].

\section{Conclusions}

The field of heavy fermions and the Kondo effect is at present very active and we have presented here three new contributions directly connected to the Kondo lattice problem. Starting from the study of the regular $S=1 / 2$ Kondo lattice model appropriate to cerium or ytterbium systems, then we present a description of the $S=1$ underscreened Kondo lattice which can account for the coexistence between the Kondo effect and the ferromagnetism observed in some uranium compounds. It yields a "Kondo-ferromagnetic diagram" given in Fig. 3 completely different from the Doniach diagram given in Fig. 1. In order to account for the pressure dependence of uranium monochalcogenides, we are developing now the new underscreened Anderson lattice model which can describe the case of a lattice of actinide or rare-earth atoms with a non-integer and variable number of $f$ electrons. Finally, we have summarized the main experimental and theoretical results obtained on the Kondo-spin glass-magnetic order competition in some disordered cerium alloys. The percolative transition observed with decreasing temperature from a paramagnetic state to a cluster glass and finally to an inhomogeneous ferromagnetic phase is a very complicated problem which needs a description of the different clusters and of their evolution with temperature.

\section{Acknowledgments}

B. Coqblin acknowledges the support of the European Cost P16 Action.

\section{References}

[1] B. Coqblin, AIP Conf. Proc. 846, 3 (2006).

[2] A.C. Hewson, The Kondo Problem to Heavy Fermions, Cambridge University Press, Cambridge 1993.

[3] B. Coqblin, J.R. Iglesias, S.G. Magalhaes, N.B. Perkins, F.M. Zimmer, J. Optoelectr. Adv. Mater. 10, 1583 (2008).

[4] A. Jayaraman, Phys. Rev. 137, A179 (1965).

[5] B. Coqblin, A. Blandin, Adv. Phys. 17, 281 (1968).

[6] R. Ramirez, L.M. Falicov, Phys. Rev. B 3, 2425 (1971). 
[7] B. Johansson, Philos. Mag. 30, 469 (1974).

[8] M. Lavagna, C. Lacroix, M. Cyrot, Phys. Lett. A 90, 210 (1982); J. Phys. F 13, 1007 (1983).

[9] J.W. Allen, R.M. Martin, Phys. Rev. Lett. 49, 1106 (1982).

[10] C.U. Segre, M. Croft, J.A. Hodges, V. Murgai, L.C. Gupta, R.D. Parks, Phys. Rev. Lett. 49, 1947 (1982).

[11] J.L. Sarrao, Physica B 259-261, 128 (1999).

[12] J. Kondo, Prog. Theor. Phys. 32, 37 (1964).

[13] J.R. Iglesias, C. Lacroix, B. Coqblin, Phys. Rev. B 56, 11820 (1997).

[14] B. Coqblin, C. Lacroix, M.A. Gusmão, J.R. Iglesias, Phys. Rev. B 67, 064417 (2003).

[15] N.B. Perkins, M.D. Nunez-Regueiro, J.R. Iglesias, B. Coqblin, Phys. Rev. B 76, 125101 (2007).

[16] S.G. Magalhaes, F.M. Zimmer, P.R. Krebs, B. Coqblin, Phys. Rev. B 74, 014427 (2006).

[17] S. Doniach, in: Proc. Valence Instabilities and Related Narrow-Band Phenomena, Ed. R.D. Parks, Plenum Press, New York 1976, p. 168.

[18] B. Barbara, H. Bartholin, D. Florence, M.F. Rossignol, E. Walker, Physica B 86-88, 177 (1977).

[19] T. Graf, J.D. Thompson, M.F. Hundley, R. Movshovich, Z. Fisk, D. Mandrus, R.A. Fischer, N.E. Phillips, Phys. Rev. Lett. 78, 3769 (1997).

[20] K. Alami-Yadri, H. Wilhelm, D. Jaccard, Physica B 259-261, 157 (1999).

[21] P. Nozières, Eur. Phys. J. B 6, 447 (1998).

[22] J. Rossat-Mignod, L.P. Regnault, J.L. Jacoud, C. Vettier, P. Lejay, J. Flouquet, E. Walker, D. Jaccard, A. Amato, J. Magn. Magn. Mater. 76-77, 376 (1988).

[23] T. Koyama, M. Nakamura, T. Mito, S. Wada, J.L. Sarrao, Phys. Rev. B 71, 184437 (2005).

[24] J. Schoenes, J. Less-Common Met. 121, 87 (1986).

[25] J. Schoenes, B. Frick, O. Vogt, Phys. Rev. B 30, 6578 (1984)

[26] Z. Bukowski, R. Troc, J. Stepien-Damm, C. Sulkowski, V.H. Tran, J. Alloys Comp. 403 , 65 (2005).

[27] V.H. Tran, R. Troc, Z. Bukowski, D. Badurski, C. Sulkowski, Phys. Rev. B 71, 094428 (2005).

[28] E. Colineau, F. Wastin, J.P. Sanchez, J. Rebizant, J. Phys., Condens. Matter 20, 075207 (2008).

[29] K.S. Burch, Elbert E.M. Chia, D. Talbayev, B.C. Sales, D. Mandrus, A.J. Taylor, R.D. Averitt, Phys. Rev. Lett. 100, 026409 (2008).
[30] Q.G. Sheng, B.R. Cooper, J. Magn. Magn. Mater. 164, 335 (1996).

[31] J. Schoenes, O. Vogt, J. Lohle, F. Hulliger, K. Mattenberger, Phys. Rev. B 53, 14987 (1996).

[32] A.L. Cornelius, J.S. Schilling, O. Vogt, K. Mattenberger, U. Benedict, J. Magn. Magn. Mater. 161, 169 (1996).

[33] P. Link, U. Benedict, J. Wittig, H. Wuhl, J. Phys., Condens. Matter 4, 5585 (1992).

[34] B. Coqblin, J.R. Iglesias, N.B. Perkins, A.S. da R. Simoes, C. Thomas, presented at the SCES 2008 Conference, Buzios, Brazil 2008.

[35] J.C. Gomez Sal, J. Garcia Soldevilla, J.A. Blanco, J.I. Espeso, J. Rodriguez Fernandez, F. Luis, F. Bartolomé, J. Bartolomé, Phys. Rev. B 56, 11741 (1997).

[36] N. Marcano, Ph.D. Thesis, Univ. of Santander, Spain 2005; N. Marcano, J.I. Espeso, J.C. Gomes Sal, J. Rodriguez Fernandez, J. Herrero-Albillos, F. Bartolome, Phys. Rev. B 71, 134401 (2005).

[37] S. Majumdar, F.V. Sampathkumaran, St. Berger, M. Della Mea, H. Michor, E. Bauer, M. Brando, J. Hemberger, A. Loidl, Solid State Commun. 121, 665 (2002).

[38] R. Vollmer, T. Pietrus, H.v. Lohneysen, R. Chau, M.B. Maple, Phys. Rev. B 61, 1218 (2000).

[39] V.S. Zapf, R.P. Dickey, E.J. Freeman, C. Sirvent, M.B. Maple, Phys. Rev. B 65, 024437 (2002).

[40] J. Garcia Soldevilla, J.C. Gomez Sal, J.A. Blanco, J.I. Espeso, J. Rodriguez Fernandez, Phys. Rev. B 61, 6821 (2000).

[41] N. Marcano, J.C. Gomez Sal, J.I. Espeso, J.M. De Teresa, P.A. Algarabel, C. Paulsen, J.R. Iglesias, Phys. Rev. Lett. 98, 166406 (2007).

[42] D. Sherrington, S. Kirkpatrick, Phys. Rev. Lett. 35, $1792(1975)$

[43] Alba Theumann, B. Coqblin, S.G. Magalhaes, A.A. Schmidt, Phys. Rev. B 63, 054409 (2001).

[44] S.G. Magalhaes, A.A. Schmidt, Alba Theumann, B. Coqblin, Eur. Phys. J. B 30, 419 (2002).

[45] S.G. Magalhaes, A.A. Schmidt, F.M. Zimmer, Alba Theumann, B. Coqblin, Eur. Phys. J. B 34, 447 (2003).

[46] D.J. Mattis, Phys. Lett. A 56, 421 (1977).

[47] F.M. Zimmer, S.G. Magalhaes, B. Coqblin, presented at the SCES 2008 Conference, Buzios, Brazil 2008. 